\title{
Uso do levantamento aéreo expedito convencional e digital para o monitoramento da cobertura florestal no Paraná: estado da arte e potencialidades
}

\author{
Fernando Luís Dlugosz ${ }^{1}$, Nelson Carlos Rosot ${ }^{1}$, Maria Augusta Doetzer Rosot², Yeda Maria Malheiros de \\ Oliveira², Marilice Cordeiro Garrastazu ${ }^{2}$
}

\begin{abstract}
${ }^{1}$ Universidade Federal do Paraná, Curso de Engenharia Florestal, Rua Lothário Meissner, 632, Jardim Botânico, CEP 80210-170, Curitiba, PR,

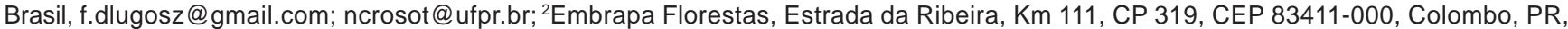
Brasil, augusta@cnpf.embrapa.br; yeda@cnpf.embrapa.br; marilice@cnpf.embrapa.br
\end{abstract}

\begin{abstract}
Resumo - O artigo apresenta uma abordagem sobre a técnica de Levantamento Aéreo Expedito no que diz respeito às suas características, aplicações e potencialidades para as condições brasileiras, principalmente no monitoramento das mudanças na cobertura florestal no Estado do Paraná. O método consiste na observação e anotação de feições ou fenômenos a partir de sobrevoos na área de interesse, voando a baixas altitudes ao longo de uma rota pré-determinada. Também são apresentadas as vantagens da introdução da sistematização digital, que definiu a nova denominação para a técnica como Levantamento Aéreo Expedito Digital. Neste caso, as anotações são realizadas digitalmente sobre tela sensível ao toque, de um computador portátil e não sobre mapa em papel, como na técnica convencional. O desenvolvimento de metodologias que demonstrem eficiência técnica e viabilidade econômica tem recebido maior ênfase em pesquisa, em função da necessidade da obtenção de informações confiáveis para subsidiar a tomada de decisões, em nível governamental, para um adequado processo de fiscalização e/ou de planejamento de uma determinada região. Considerando a dinâmica de uso da terra e a necessidade de se monitorar a cobertura vegetal, pode-se afirmar que o Levantamento Aéreo Expedito apresenta elevado potencial de aplicação às condições brasileiras e ainda ótima relação custo-benefício.
\end{abstract}

Termos para indexação: Levantamento aéreo, geoprocessamento, uso da terra.

\section{Conventional aerial sketchmapping and digital aerial sketchmapping development for forest monitoring in Paraná: state of art and potentialities}

\begin{abstract}
This paper presents an approach to the technique of aerial sketchmapping in respect to its characteristics, applications and potential for use in Brazil, mainly to monitor changes in forest cover in Paraná state. The method consists of observation and annotation of features or phenomena from overflights in the area of interest flying at low altitudes along a predetermined route. It also presents the advantages obtained with the introduction of digital aerial sketchmapping, which defined the new name for the technique as Digital Aerial Sketchmapping. In this case the annotations are performed digitally on the screen (touch screen) of a notebook and not on paper map, as in the conventional technique. The development of methodologies that demonstrate technical efficiency and economic viability has received increased emphasis on research, according to the necessity of obtaining reliable information to support decision making at the government level, for an appropriate monitoring procedure and/ or planning a given region. Considering the dynamics of land use and the need to monitor land cover, it can be stated that the Aerial Sketchmapping has high potential for application to brazilian conditions and still excelent cost/benefit relation.
\end{abstract}

Index terms: Aerial survey, geoprocessing, land use.

\section{Introdução}

Uma das prioridades na tomada de decisões, em nível governamental, para um adequado processo de fiscalização da vegetação e/ou de planejamento de uma determinada região, diz respeito à disponibilidade de levantamentos temáticos, que mostrem o estado atual dos recursos naturais e seu uso pelo homem. Neste contexto, é de suma importância tornar operacional e de acesso público a cartografia temática das áreas desflorestadas, 
em uma base de dados, tanto para os tomadores de decisões, quanto para aqueles interessados em modelar os efeitos dos processos antrópicos (Duarte et al., 1999).

De acordo com Oliveira et al. (2007), considerando-se a importância dos recursos naturais para a regulação da vida no planeta, há a necessidade de aplicação de limites a cada atividade antrópica, visando dirimir resultados negativos sobre a qualidade do meio ambiente. Uma forma de atuação sobre as alterações regionais, possibilitando o acompanhamento de mudanças, é o desenvolvimento e adoção de técnicas de monitoramento do uso da terra, em nível espacial e temporal.

A Declaração de Santiago define monitoramento como sendo a medição e avaliação periódica e sistemática da mudança em um indicador, mais especificamente, "a aferição regular que tem como objetivo determinar a ocorrência de desvios com relação a uma norma esperada” (Estraviz-Rodriguez, 1998).

Os estados brasileiros necessitam de monitoramento das mudanças no uso da terra e suas consequências sobre a cobertura florestal. Como exemplo, a contratação em 2005 de um projeto de atualização do mapeamento sistemático do Estado do Paraná, em escala 1:50.000, revela o interesse e a preocupação do governo em estabelecer uma linha de base relativa à atual situação dos remanescentes florestais e demais usos da terra, para, a partir dela, poder desenvolver um sistema de monitoramento contínuo do uso da terra. Desta forma, busca-se proporcionar ferramental para o estudo de mudanças ao longo do tempo, além de subsidiar decisões e ações de planejamento e administração da ocupação ordenada e racional do meio físico.

A observação temporal de determinadas características da paisagem é a base para o monitoramento ambiental (Mace, 1997). Um sistema de monitoramento de abrangência regional ou estadual deve prever em sua estruturação alguns componentes estratégicos que aliem a capacidade de verificar grandes áreas à garantia de obtenção do nível de detalhamento desejado para a variável de interesse. Em especial, no que diz respeito aos sistemas de monitoramento da vegetação, acreditase que as técnicas de levantamento aéreo - quer sejam associadas à aquisição de imagens ou não - podem vir a contribuir no sentido de se estabelecer um compromisso entre área sobrevoada e escala de levantamento, envolvendo para tanto os indicadores para recursos ambientais selecionados para cada processo (Oliveira et al., 2007).
A técnica de Levantamento Aéreo Expedito, conhecida nos Estados Unidos como Aerial Sketchmapping, é uma ferramenta de sensoriamento remoto, usada para a avaliação das condições fitossanitárias das florestas da América do Norte (Ciesla, 2000). A partir de 2001, foi adaptada às condições brasileiras através de parceria entre a Embrapa Florestas e USDA Forest Service, com o objetivo inicial de monitorar a vespa-damadeira (Sirex noctilio) em plantações de Pinus spp. no sul do país (Oliveira et al., 2008). A técnica - que permite o monitoramento das condições da floresta - é relativamente simples e de baixo custo. Esta consiste na observação e anotação de feições ou fenômenos a partir de sobrevoos na área de interesse, utilizando aviões de asa alta, voando a baixas altitudes ao longo de uma rota pré-determinada. Com a introdução da sistematização digital, que definiu a nova denominação para a técnica como Levantamento Aéreo Expedito Digital, as anotações são realizadas digitalmente sobre a tela sensível ao toque de um notebook e não sobre mapa em papel, como na técnica convencional.

Um processo de treinamento e várias campanhas de campo e aéreas foram realizados ao longo dos anos subsequentes, tornando a técnica conhecida e criando possibilidades de sua utilização em distintas ocasiões. Adicionalmente, as técnicas de levantamento aéreo expedito, tanto convencional (LAE) quanto digital (LAED) promovem a integração das geotecnologias como o Sensoriamento Remoto (SR), o Global Navigation Satellite System (GNSS) e Sistemas de Informações Geográficas (SIG), necessárias nos levantamentos e estudos dos recursos naturais, que visam produzir soluções para o acompanhamento das mudanças no uso/cobertura da terra ao longo do tempo.

Desta forma, o presente trabalho apresenta as características, o estado-da-arte, bem como as potencialidades do Levantamento Aéreo Expedito Convencional, como ferramenta para o monitoramento das mudanças na cobertura florestal, e seu sucessor, o Levantamento Aéreo Expedito Digital, com tecnologia embarcada.

\section{Descrição da Técnica de LAE}

Para a realização de estudos usando LAE, algumas diretrizes técnicas devem ser consideradas, sendo mais relevantes as características da aeronave, o perfil do observador aéreo e a assinatura do dano ou feição de interesse. 
A aeronave deve possuir janelas grandes, que permitam maior visibilidade, pois o LAE depende basicamente da anotação feita pelos observadores aéreos. A utilização de aviões de asa alta colaboram para esse fator, por não proporcionar o efeito conhecido como "zona cega", causado em aviões de asa baixa, que pode impedir ou dificultar a observação da feição desejada no momento exato em que a aeronave a sobrevoa. O uso de helicópteros seria uma excelente alternativa, porém o custo de sua operação chega a ser três vezes mais caro que aqueles das aeronaves com asas fixas (Ciesla, 2000).

A velocidade de deslocamento deve ser entre 90-100 Mph (145-160 km h $\mathrm{h}^{-1}$ ), sendo que velocidades maiores não são condizentes com o tipo de trabalho por dificultar a anotação das informações observadas. A altura de voo pode variar de 1.000-1.500 pés (300-450 m) sobre elevação média do terreno. Recomenda-se não realizar levantamentos aéreos por mais de seis horas por dia devido à fadiga e à tensão, tanto do piloto como dos observadores, assim como não iniciar os voos antes das 8 horas da manhã, em função da possível presença de sombras nas faces oeste e sul das escarpas e pela possibilidade de ocorrência de neblina matinal (Oliveira et al., 2008).

O treinamento do observador é fundamental para a qualidade do levantamento, sendo que ele deve possuir amplo conhecimento do objetivo da missão, das características dos alvos a serem abordados e possíveis variações de aparência dentro da mesma classe, que pode ser influenciada pela sua fenologia, estágio de desenvolvimento ou da época de análise. Essa capacitação permitirá discriminação de áreas ou feições, para então "desenhar" esses pontos ou polígonos sobre um mapa-base, identificando-os de acordo com um código pré-estabelecido. Segundo Ciesla (2000), algumas das características que determinam a assinatura de um objeto observado à distância e que permitem que ele seja identificado são: cor ou coloração, forma, brilho, textura e posição espacial. A realização de missões continuadas para o monitoramento de um mesmo conjunto de variáveis ou eventos permite que os observadores passem a construir e a elaborar padrões para as "assinaturas" necessárias a determinado objetivo.

As "assinaturas de feições" ou "assinaturas de danos" podem ser descritas como uma combinação de características que permitem ao observador aéreo identificar determinados aspectos da variável de interesse ou dos sintomas associados a agentes específicos, causadores de danos florestais, na forma como aparecem a partir de uma perspectiva aérea. Ainda, os observadores envolvidos em uma campanha devem apresentar uma sistematização padronizada de avaliação e anotação, de maneira a evitar confusão de interpretação e erros nos resultados. Deve-se enfatizar que o sucesso de uma campanha aérea é alcançado pelo esforço não somente dos observadores aéreos, mas sim de toda a equipe como pilotos, pessoal de apoio terrestre, além de todos os envolvidos no planejamento, digitalização e compilação da base de dados.

Com relação aos danos florestais, os resultados obtidos pela técnica podem ser utilizados para: a) monitorar danos causados por pragas; b) iniciar operações de corte preventivo em povoamentos infestados; c) planejar operações de manejo de pragas; e d) estruturar um banco de dados históricos relativos à ocorrência e intensidade de infestações por insetos ou doenças (Ciesla et al., 2002). Além destas, o LAE também foi empregado na detecção e mapeamento de incêndios e danos causados por eventos naturais. Também apresenta grande potencial de uso no mapeamento de regiões que sofreram mudanças na cobertura da terra, principalmente por desmatamento, o que vem sendo apontado como um fator de grande influência sobre as mudanças do clima, além de perda da diversidade biológica.

Conforme Estados Unidos (2005), o Levantamento Aéreo Expedito (LAE), dito convencional, é executado usando mapas impressos em papel. Com os avanços computacionais e da tecnologia de telas sensíveis aoi toque tornou-se possível capturar as observações aéreas diretamente em formato digital compatível com uma base de dados de Sistema de Informações Geográficas, dinamizando, deste modo, o levantamento, a conversão e o processamento de dados. Essa evolução tecnológica deu origem a uma nova fase do Levantamento Aéreo Expedito, denominada originalmente de Digital Aerial Sketchmapping (D-ASM) e traduzida como Levantamento Aéreo Expedito Digital (LAED).

Assim, desde o início do século 21, o USDA Forest Service, através de sua equipe de Tecnologia em Sanidade (Forest Health Technology Enterprise Team - FHTET) e do Centro de Aplicações de Sensoriamento Remoto (RSAC), têm trabalhado em conjunto para desenvolver o LAED (Ciesla, 2008). Inicialmente, o sistema foi desenvolvido usando computadores portáteis (notebooks) comercialmente 
disponíveis (Schrader-Patton, 2008), sendo que para um segundo observador poderia ser eventualmente utilizado mais um monitor do tipo tablet PC acoplado ao notebook principal.

O Levantamento Aéreo Expedito Digital (LAED), que utiliza computadores acoplados a um equipamento de posicionamento global (GPS), vem substituindo os procedimentos que utilizam mapas em meio analógico. Os dados gravados podem ser incorporados a um SIG, sem os passos de digitalização utilizados no esquema anterior (LAE), que consumiam significativo tempo para execução.

O núcleo do sistema LAED é o programa GeoLink, desenvolvido pela empresa Michael J. Baker Incorporation. O GeoLink incorpora o sinal de GPS, mostrado em janela exibida na tela (Figura 1) e permite que o observador aéreo desenhe pontos, linhas ou polígonos sobre mapas previamente elaborados e disponibilizados no sistema, acompanhando, durante o voo planejado, a feição ou evento que acontece em terra. A tela do sistema mostra a localização da aeronave no mapa do levantamento, eliminando as fontes de erros relacionadas a problemas de localização dos observadores (em relação ao mapa), quando em voo (Ciesla, 2008).

Entretanto, os conceitos básicos do LAE convencional aplicam-se igualmente ao digital. Para Ciesla (2008), contribuíram para tal evolução tecnológica: a) os avanços na velocidade dos microprocessadores e nos componentes dos computadores do tipo PC; b) a melhoria de desempenho dos sistemas operacionais; c) a popularização do uso do GPS; e d) o desenvolvimento de tecnologia envolvendo monitores sensíveis ao toque e que permitem leitura de tela quando em exposição à luz solar, tornaram possível o uso de notebooks para registro de dados em levantamentos aéreos.

A partir do desenvolvimento inicial do sistema, o LAED original foi apresentado para tripulações americanas que trabalham no monitoramento da vida selvagem. O conceito foi aprovado, mas foi demandado o desenvolvimento de sistemas fisicamente menores, que pudessem incluir um meio de obtenção da informação e possibilidade de anotação de ocorrências ou fenômenos em tempo real. Assim, um tablet PC foi selecionado, possuindo este uma caneta óptica (digital) acoplada e um sistema de transferência via Internet de comunicação remota desenvolvido pela Nasa-Goddard (SchraderPatton, 2008).

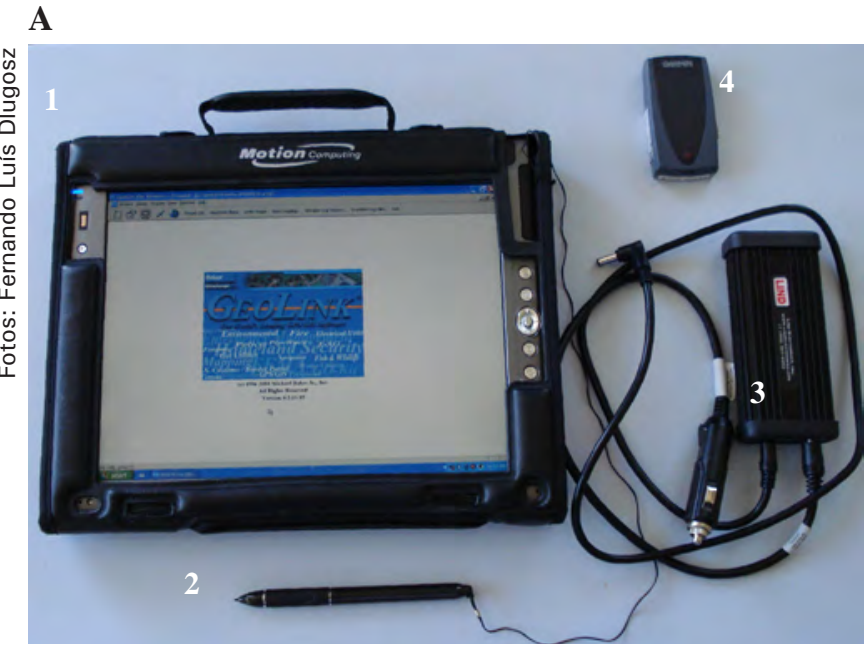

B

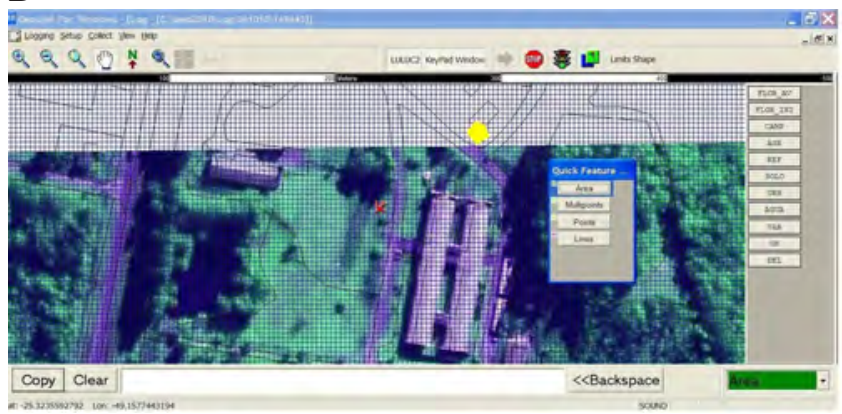

Figura 1. Equipamentos e sistema informatizado utilizados a bordo no LAED, sendo: (A) tablet PC (1); caneta óptica (2); alimentador via tomada 12V (3); GPS Garmin 10 com transmissão de dados por sistema Bluetooth (4); e (B) Interface do programa GeoLink.

\section{Aplicações de LAE e LAED no monitoramento florestal}

Ciesla (2008) comenta que, apesar da introdução de novas tecnologias de detecção remota, o Aerial Sketchmapping é, ainda, a principal técnica na avaliação de danos florestais causados por insetos, doenças e por outros agentes. Esta técnica forneceu dados sobre posição e intensidade de manifestações de pragas desde o final dos anos 1940. Atualmente, o LAE é considerado como operação de rotina para a detecção de pragas florestais nos EUA e Canadá. Anualmente, são percorridos por meio de LAE em média 9 milhões de hectares, em aproximadamente 300 horas de voo, com de $32 \mathrm{mil}$ hectares sobrevoados por hora (Oliveira et al., 2007). Também, na Austrália, são relatados usos da técnica para detecção e avaliação de ataques de pragas em plantações de espécies dos gêneros Pinus e Eucalyptus. 
No Brasil, Oliveira et al. (2004) descrevem os procedimentos do uso do LAE para o mapeamento de danos fitossanitários em povoamentos florestais no Estado do Paraná. Os trabalhos foram conduzidos pela Embrapa Florestas e USDA-FS na região de União da Vitória e Pitanga, PR, sendo realizados em três campanhas de voos operacionais nos anos de 2002, 2003 e 2004, perfazendo mais de 1 milhão de hectares, que correspondem a aproximadamente $5 \%$ da área total do estado. Todos os levantamentos foram efetuados utilizando o método do voo em grade, com linhas de voo definidas em intervalos de $4 \mathrm{~km}$, determinando uma faixa de mapeamento de $2 \mathrm{~km}$ para cada observador. Foram também realizados voos de treinamento para discriminação de diferentes fases sucessionais em remanescentes de Floresta com Araucária e levantamentos para monitorar desmatamentos nesse ecossistema.

Quando a equipe da Embrapa Florestas optou por produzir cartas-imagem como base para o LAE, constatou-se que os voos poderiam servir como meio de verificação da acuracidade temática de mapeamentos já existentes, elaborados por meio de outras técnicas. Desta forma, a técnica foi testada com esta nova finalidade, sendo aprovada (Oliveira et al., 2004), já que além da boa relação custo-benefício, representa uma possibilidade real de integração a um sistema mais amplo de monitoramento ambiental. Os resultados obtidos com as campanhas realizadas ao longo dos últimos anos levaram à sua proposição como alternativa para a avaliação da acuracidade de mapeamentos realizados em nível regional ou nacional, como no caso das unidades amostrais de paisagem do Inventário Florestal Nacional do Brasil, cujo projeto está em execução através de um acordo de cooperação técnica entre o Serviço Florestal Brasileiro e a FAO (Oliveira et al., 2009).

Além dos trabalhos da equipe da Embrapa Florestas, Santos \& Pabis (2008) utilizaram o Levantamento Aéreo Expedito, associado à tomada de fotografias aéreas inclinadas, para o apoio ao desenvolvimento do inventário florestal quali-quantitativo, sendo que 70\% das parcelas lançadas em campo foram baseadas nas associações mapa-foto. Os autores comentam, ainda, que o uso do LAE norteou ações de adequação do uso da terra, direcionamento das equipes de proteção florestal e patrimonial, além do planejamento silvicultural.

Schrader-Patton (2003) apresenta uma das primeiras publicações envolvendo o LAED. Nesta, foi descrito o projeto do desenvolvimento do sistema, que possuía o objetivo de substituir ou maximizar a eficiência do sistema LAE. Para tanto, vários equipamentos e opções de programas foram investigados. Este sistema apresentou como indicadores favoráveis o rastreamento automático da posição da aeronave na base cartográfica por meio da conexão a um receptor GPS e a redução do tempo de digitalização de dados em um SIG. O autor previa melhorias futuras para o sistema, incluindo modificações no programa para a interface do usuário, o desenvolvimento de um sistema de entrada dupla e ampliação dos dados utilizados como mapas de fundo.

Karl \& Porter (2006) realizaram levantamentos por meio da técnica de LAED na área do Rio das Serpentes do Hells Canyon, em Idaho-Oregon (EUA), no ano de 2004. $\mathrm{O}$ objetivo do trabalho foi a detecção e mapeamento de plantas invasoras, sendo, neste caso, realizado por meio de helicóptero que permitia voos mais lentos e em menores alturas. As campanhas foram realizadas entre maio e outubro (próximo do verão nos EUA), tendo cada campanha foco preliminar em determinada espécie invasora, mas anotando também dados de outras espécies possíveis de serem identificadas. Os autores mencionam que, para melhorar a localização dos alvos e aumentar a eficiência do inventário, é necessário segmentar a área em subunidades, dividindo-a com base em características facilmente reconhecidas do ar. Porém, para cada subdivisão da área, os mesmos critérios devem ser utilizados nas avaliações subsequentes para evitar inconsistências. Como desvantagem do emprego da técnica, apontaram dificuldades na observação de indivíduos pequenos ou isolados.

Conforme apresentado em Cooperative Invasive Species Management Area (2009), foi iniciado em 2005, no estado da Flórida (EUA), o projeto piloto desenvolvido através de convênio de cooperação entre o Distrito de Gerenciamento de Água do Sul da Flórida (South Florida Water Management District SFWMD) e o Serviço Nacional de Parques (National Park Service - NPS), que tinha por objetivo avaliar, por meio de Levantamento Aéreo Expedito Digital, a distribuição espacial e a abundância de sete espécies invasoras que ocorriam em Unidades de Conservação, sendo elas: Lygodium microphyllum, Melaleuca quinquenervia, Schinus terebinthifolius, Pennisetum purpureum, Neyraudia reynaudiana, Casuarina equisetifolia e Thespesia populnea. Foram sobrevoadas a Reserva Natural Nacional de Refúgio da Vida 
Silvestre de Loxahatchee, as Áreas de Conservação de Água (WCA-2, WCA-3A e WCA-3B) e o Parque Nacional de Everglades. O projeto apresentou resultados satisfatórios, fato que, em 2008, trouxe a recomendação de avaliações bianuais em todas as áreas que estão sob gerência da Cooperative Invasive Species Management Area, que representa 1,09 milhão de hectares. As faixas de voo foram de $1 \mathrm{~km}$ de largura, sendo sobrevoadas em sentido leste-oeste, com velocidade de 130 a $160 \mathrm{~km} \mathrm{~h}^{-1}$ em altura de aproximadamente $150 \mathrm{~m}$ acima do nível do solo. Os polígonos foram gerados por espécie avaliada, agrupando-se em classes conforme as densidades regionais encontradas, sendo: alta densidade (maior que $50 \%$ ); média densidade (entre $25 \%$ e $50 \%$ ) e baixa densidade (menor que 25\%).

Hinkley et al. (2006) apresentaram a sistematização desenvolvida pelo Centro de Aplicações de Detecção Remota (RSAC) do Serviço Florestal Americano em parceria com a Nasa-Goddard Space Flight Center para uso na detecção e registro de incêndios. O sistema, que integra a técnica de LAED a uma fonte de comunicação remota de longo alcance para repasse de dados com alta velocidade denominado de Remote Internet Protocol Communications System (RIPCom), permite aos peritos em combate a incêndios coletar informações pertinentes ao incidente (como perímetro do incêndio, pontos de calor, estruturas existentes e rotas de acesso) e disseminar de forma rápida estas informações ao comando de combate e a outras frentes de operação.

Um projeto realizado em conjunto pela Universidade Federal do Paraná e Embrapa Florestas propôs a definição de um sistema de monitoramento e verificação da acuracidade de mapeamento temático empregando a técnica de LAED, por meio de amostragem sistemática em faixas. A área piloto contemplou a abrangência da carta planialtimétrica denominada Campo Largo, elaborada pela Diretoria de Serviço Geográfico do Exército (DSG). A referida área encontra-se entre as coordenadas $25^{\circ} 00^{\prime}$ e $25^{\circ} 15^{\prime}$ de latitude sul (extensão de 25,20 km) e 49 $30^{\prime}$ ' $49^{\circ} 45^{\prime}$ de longitude oeste (extensão de $27,64 \mathrm{~km}$ ), contemplando parcialmente o território dos municípios de Campo Largo, Campo Magro e Balsa Nova, totalizando $696,58 \mathrm{~km}^{2}$. Nesta, engloba-se parte da Área de Proteção Ambiental da Escarpa Devoniana e seu entorno, o que exige o controle de atividades antrópicas sujeitas ao cumprimento de determinadas restrições legais. A necessidade de monitoramento associada às características de relevo, que dificulta $o$ controle via terrestre, foram tomadas como relevantes para a escolha da área.

\section{Viabilidade da Técnica}

Levantamentos aéreos são reconhecidamente um poderoso aliado do monitoramento, em função da rapidez na obtenção de dados. Este é um dos mais fortes argumentos para a adoção do LAE ou LAED para o monitoramento, principalmente, os que envolvem grandes áreas. Além disso, o acesso para coleta de dados do objeto de interesse, quando usada a técnica de LAE ou LAED, não apresenta restrições no que se refere ao uso de estradas e caminhos, sendo possível acessar, facilmente, áreas longínquas e até mesmo inóspitas.

O monitoramento aéreo possui uma ótima relação custo-benefício, já que cobre extensas áreas florestais, a maioria delas inacessíveis, em um tempo relativamente curto. Considerando-se o tempo de espera com relação às condições climáticas, pode-se afirmar que 200 mil hectares por dia podem ser monitorados confortavelmente por uma equipe de dois observadores experientes. Para uma velocidade de $145-190 \mathrm{~km} / \mathrm{h}$, a capacidade de se observar chega a 256 ha a cada 15 segundos de voo. Na Tabela 1 apresenta-se um comparativo entre levantamentos terrestres e o LAE para a detecção de pragas em povoamentos de Pinus spp.

Tabela 1. Comparativos entre grandezas envolvidas nos monitoramentos aéreo expedito e terrestre.

\begin{tabular}{lcc}
\hline \multicolumn{1}{c}{ Monitoramento } & LAE & Terrestre \\
\hline Custo ha $^{-1}$ & ${\text { US\$ } 0,02^{(1)} \mathrm{a}}^{(2)}$ & US\$ 5,26 \\
Tempo & US $0,33^{(2)}$ & 86,4 mil horas \\
Equipe & 1 hora & 3 pessoas \\
\hline
\end{tabular}

Fonte: Adaptado de Oliveira et al. (2008).

(1) Para uma área maior que $300 \mathrm{mil} \mathrm{ha;}{ }^{(2)}$ Para uma área de aproximadamente 4 mil ha; ${ }^{(3)} 1$ Piloto e dois técnicos.

Segundo Cooperative Invasive Species Management Area (2009), o custo efetivo para a execução do levantamento foi de US\$ 0,09 por hectare, na avaliação de espécies invasoras. Também, segundo Karl \& Porter (2006), em trabalho similar de inventário de espécies invasoras, o custo variou de US\$ 0,27 a US\$ 1,75 por hectare avaliado, sendo, neste caso, utilizado um helicóptero, fato que evidencia o maior custo, como já discutido anteriormente. 
Devem-se prever possíveis variações no custo devido à influência do tempo de voo do aeroporto até a área de estudo, habilidades do observador aéreo, objetivos da missão, características da região sob análise e da aeronave. Estes fatores podem, separadamente ou em conjunto, exercer considerável alteração nos valores do levantamento. Vale ressaltar a importância de se contabilizar os custos para treinamento de observadores aéreos, bem como sua remuneração e de outros técnicos que atuam na compilação da base de dados para o levantamento. Ainda, na fase de planejamento, sempre deverá ser considerada a possibilidade de mau tempo, que é um potencial problema causador de atrasos nos aerolevantamentos.

Segundo Schrader-Patton (2008), os custos de aquisição, nos EUA, de equipamento como notebooks, tablet touchscreen, ou pen tablet, GPS e cabos de conexão podem variar de US\$ $\$ .000,00$ a US\$10.000,00, dependendo da configuração escolhida. Para aquisição do software GeoLink o custo é de aproximadamente US\$ $1.400,00$. Também a compra e o processamento dos dados digitais de base ou auxiliares devem ser considerados no orçamento, uma vez que variam em função do objetivo do trabalho e da disponibilidade.

\section{Considerações finais}

Em função da necessidade de se aprimorar os métodos de monitoramento dos recursos naturais, incluindo-se a produção de bases confiáveis, pode se afirmar que a utilização da técnica de Levantamento Aéreo Expedito, tanto convencional quanto digital, representa uma alternativa potencial para tal propósito, apresentando-se adequada em regiões com grandes extensões de área e com dificuldade de acesso terrestre.

Possibilita, ainda, a obtenção de informações adicionais, tais como intensidade, extensão e potencial aumento de danos florestais, não se restringindo à simples presença ou ausência de um fato ou sua posição. Além disto, ações por terra para autuações e embargos de atividades antrópicas irregulares ou ilegais podem acontecer simultaneamente ao voo, com a transmissão das coordenadas observadas no GPS em tempo real via rádio ou após o processamento dos dados em ambiente SIG em tempo relativamente curto.

A grande vantagem da técnica digital consiste na captura das observações aéreas diretamente em formato compatível com uma base de dados de Sistema de Informações Geográficas, dinamizando, deste modo, o levantamento, a conversão e o processamento dos dados.

O avanço no processo de adaptação do LAED, principalmente no que diz respeito ao uso do equipamento, à sistematização dos procedimentos para captação dos dados durante o voo, à definição de uma sequência de operações pós-voo para processamento dos dados e à execução de análises relativas aos objetivos do levantamento, permitirá o futuro estabelecimento de um sistema contínuo de monitoramento da cobertura e uso da terra, incluindo danos causados às florestas.

\section{Referências}

CIESLA, W. M. Remote sensing in forest health protection. Salt Lake City: USDA, Forest Service, Remote Sensing Applications Center; Fort Collins: USDA, Forest Service, Forest Health Technology Enterprise Team, 2000. 266 p. (FHTET Rep., 00-03).

CIESLA, W. M.; JOHNSON, E. W.; OLIVEIRA, Y. M. M de; ROSOT, M. A. D.; ELLENWOOD, J.; PENTEADO JÚNIOR, J. F. Development of an aerial sketchmap program for detection and mapping of forest damage in Brazil. In: SEMINÁRIO DE ATUALIZAÇÃO: SENSORIAMENTO REMOTO E SISTEMAS DE INFORMAÇÕES GEOGRÁFICAS APLICADOS À ENGENHARIA FLORESTAL, 5., 2002, Curitiba. Anais. Curitiba: [s.n., 2002]. p. 31-38. Ed. por Attilio Antonio Disperati: João Roberto dos Santos

CIESLA, W. Technologies and Observer Training for Improved Aerial Forest Health Surveys in the United States. In: SEMINÁRIO DE ATUALIZAÇÃO EM SENSORIAMENTO REMOTO E SISTEMAS DE INFORMAÇÕES GEOGRÁFICAS APLICADOS À ENGENHARIA FLORESTAL, 8, 2008, Curitiba. Anais. Curitiba: FUPEF, 2008, p. 723-728. Ed. por Attilio Antonio Disperati: João Roberto dos Santos.

COOPERATIVE INVASIVE SPECIES MANAGEMENT AREA (CISMA). Everglades CISMA Digital Aerial Sketchmapping (DASM). Florida: University of Georgia, Center for Invasive Species and Ecosystem Health, 2009. Disponível em: $<$ http:// www.evergladescisma.org/DASM/>. Acesso em: 12 abr. 2010.

DUARTE V.; SHIMABUKURO, Y. E.; SANTOS, J. R. dos; MELLO, E. M. K.; MOREIRA, J.C.; MOREIRA, M. A.; SOUZA, R. C. M. de; SHIMABUKURO, R. M. K.; FREITAS, U. M. de. Metodologia para criacao do PRODES digital e do banco de dados digitais da Amazonia - Projeto BADDAM. São José dos Campos: INPE, 1999. 33p. (INPE. 7032-PUD/035).

ESTADOS UNIDOS. Department of Agriculture. Forest Service. Aerial Survey Geographic Information System Handbook: Sketchmaps to Digital Geographic Information. Colorado: USDA. Forest Service, 2005. 35 p. Disponível: <http://www. fs.fed.us/foresthealth/technology/pdfs/GISHandbook_body_ apndxA-C.pdf > . Acesso em: 30 maio 2009. 
ESTRAVIZ-RODRIGUEZ, L. C. Monitoramento florestal: iniciativas, definições e recomendações. Série Técnica IPEF, Piracicaba, v. 12, n. 31, p. 9-21, abr. 1998. Workshop sobre Monitoramento Ambiental em Áreas Florestadas, 2., 1997, Piracicaba. Memória.

HINKLEY, E.; ZAJKOWSKI, T.; SCHRADER-PATTON, C. Digital Aerial Sketchmapping. Forest Encyclopedia Network. South Salt Lake City: USDA Forest Service, 2006. Disponível em: <http://www. forestencyclopedia.net/p/p62>. Acesso em: 02 abr. 2010.

KARL, J. W.; PORTER, M. Digital Aerial Sketch-Mapping for Early Detection and Mapping. In: REW, L. J.; POKORNY, M. L. (Eds.). Inventory and Survey Methods for Nonindigenous Plant Species. Bozeman: Monana State University Extension, 2006, cap. 4, p. 33-41. Disponível em: <http://www.cof.orst.edu/cof/teach/ fs548/pdfs/Wk3Inventory.pdf $>$. Acesso em: 02 abr. 2010.

MACE, T. H. Environmental monitoring. In: AMERICAN SOCIETY FOR PHOTOGRAMMETRY AND REMOTE SENSING. Manual of photographic interpretation.

Maryland: American Society for Photogrammetry and Remote Sensing, 2 ed., 1997. cap. 17, p. 591-612.

OLIVEIRA, Y. M. M. de; GARRASTAZU, M. C.; ROSOT, M. A. D.; SOARES, A. de O.; PENTEADO JÚNIOR, J. F. Levantamento Aéreo Expedito (LAE). Colombo: Embrapa Florestas, 2007. (Embrapa Florestas. Documentos, 157). 1 CDROM.

OLIVEIRA, Y. M. M. de; GARRASTAZU, M. C.; ROSOT, N. C.; ROSOT, M. A. D.; SOARES, A. de O.; PENTEADO JÚNIOR, J. F.; CIESLA, W. M.; JOHNSON, E. Levantamento Aéreo Expedito (LAE). 2. ed. rev. Colombo: Embrapa Florestas, 2008. 53 p. (Embrapa Florestas. Documentos, 157).
OLIVEIRA, Y. M. M. de; ROSOT, M. A. D.; CIESSLA, W. M.; JOHNSON, E. W.; RHEA, R.; PENTEADO JUNIOR, J. F.; LUZ, N. B. da. O mapeamento aéreo expedito para o monitoramento florestal no sul do Brasil. In: DISPERATI, A. A.; SANTOS, J. R. dos (Ed.). Aplicações de geotecnologias na engenharia florestal. Curitiba: Copiadora Gabardo, 2004. p. 12-24. OLIVEIRA, Y. M. M. de; ROSOT, M. A. D.; GARRASTAZU, M. C.; JUNIOR, J. F. P.; MATTOS, P. P. de; LACERDA, A. E. B. de; PONZONI, F. J.; FREITAS, J. V. de; GOMIDE, L. A.; CIESLA, W. Evaluación de la acuracidade del mapeo de la vegetación del Inventario Forestal Nacional de Brasil. In: CONGRESO FORESTAL MUNDIAL, 13., 2009, Buenos Aires. Desarrollo forestal: equilibrio vital. Argentina: FAO, 2009. Resumo. Disponível em: <http://www.cfm2009.org/es/ programapost/trabajos/Accuracy_assessment_Brazilian_National_ FD.pdf $>$ Acesso em: 12 mar. 2010.

SANTOS, A. L. dos; PABIS, L. Levantamento aéreo expedito para inventário florestal de reconhecimento. In: SEMINÁRIO DE ATUALIZAÇÃO EM SENSORIAMENTO REMOTO E SISTEMAS DE INFORMAÇÕES GEOGRÁFICAS APLICADOS À ENGENHARIA FLORESTAL, 8., 2008, Curitiba. [Anais]. [Curitiba]: FUPEF, 2008. p. 365-370.

SCHRADER-PATTON, C. Digital aerial sketchmapping. USDA Forest Service, Remote Sensing Applications Center, Salt Lake City, UT and Forest Health Technology Enterprize Team, 2003, 17 p. (Fort Collins, CO, Report RSAC 1202-RPT2).

SCHRADER-PATTON, C. Digital Aerial Sketchmapping (DASM) for invasive weeds. In: A Weed Manager's Guide to Remote Sensing and GIS - Mapping \& Monitoring. USDA Forest Service Remote Sensing Applications Center (RSAC), 2008. Disponível em: <http://www.fs.fed.us/eng/rsac/ invasivespecies/documents/DASM.pdf> Acesso em: 12. jan. 2009.

Recebido em 17 de julho de 2010 e aprovado em 08 de novembro de 2010 